\title{
MOAO first on-sky demonstration with CANARY
}

\author{
E. Gendron ${ }^{1}$, F. Vidal ${ }^{1}$, M. Brangier ${ }^{1}$, T. Morris ${ }^{2}$, Z. Hubert ${ }^{1}$, A. Basden ${ }^{2}$, G. Rousset ${ }^{1}$, R. Myers ${ }^{2}$, \\ F. Chemla ${ }^{3}$, A. Longmore ${ }^{4}$, T. Butterley ${ }^{2}$, N. Dipper ${ }^{2}$, C. Dunlop ${ }^{2}$, D. Geng ${ }^{2}$, D. Gratadour ${ }^{1}$, D. Henry ${ }^{4}$,

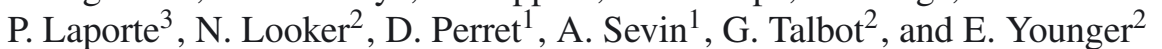 \\ ${ }^{1}$ LESIA, Observatoire de Paris, CNRS, UPMC, Université Paris Diderot, 5 place J. Janssen, 92190 Meudon, France \\ e-mail: firstname.lastname@obspm.fr \\ 2 Centre for Advanced Instrumentation, Durham University, South Road, Durham DH1 3LE, UK \\ 3 GEPI, Observatoire de Paris, CNRS, Université Paris Diderot, 5 place J. Janssen, 92190 Meudon, France \\ ${ }^{4}$ UKATC, Royal Observatory Edinburgh, Blackford Hill, Edinburgh EH9 3HJ, UK
}

Received 4 February 2011 / Accepted 23 February 2011

ABSTRACT

\begin{abstract}
Context. A new challenging adaptive optics (AO) system, called multi-object adaptive optics (MOAO), has been successfully demonstrated on-sky for the first time at the $4.2 \mathrm{~m}$ William Herschel Telescope, Canary Islands, Spain, at the end of September 2010.

Aims. This system, called CANARY, is aimed at demonstrating the feasibility of MOAO in preparation of a future multi-object near infra-red (IR) integral field unit spectrograph to equip extremely large telescopes for analysing the morphology and dynamics of high-z galaxies.

Methods. CANARY compensates for the atmospheric turbulence with a deformable mirror driven in open-loop and controlled through a tomographic reconstruction by three widely separated off-axis natural guide star (NGS) wavefront sensors, which are in open loop too. We compared the performance of conventional closed-loop AO, MOAO, and ground-layer adaptive optics (GLAO) by analysing both IR images and simultaneous wave-front measurements.

Results. In $H$-band, Strehl ratios of 0.20 are measured with MOAO while achieving 0.25 with closed-loop AO in fairly similar seeing conditions $\left(r_{0} \approx 15 \mathrm{~cm}\right.$ at $\left.0.5 \mu \mathrm{m}\right)$. As expected, MOAO has performed at an intermediate level between GLAO and closed-loop AO.
\end{abstract}

Key words. instrumentation: adaptive optics - instrumentation: high angular resolution - atmospheric effects - galaxies: formation - galaxies: high-redshift

\section{Introduction}

Adaptive optics $(\mathrm{AO})$ is a technique that compensates in real time with a deformable mirror (DM) the wavefront of the incoming stellar light, which has been distorted by the Earth's atmosphere. AO became available for astronomers at the end of the 1980's (Rousset et al. 1990), and its success is such that the next generation of giant telescopes of 20-40 m class cannot be conceived without it (Clénet et al. 2010). For future astrophysical programmes, new concepts are needed to fulfil the requirements for wide-field observation, such as the ground layer AO (GLAO) (Milton et al. 2008) or the multiconjugate AO (MCAO) (Marchetti et al. 2008). Amongst the various concepts, multi-object adaptive optics (MOAO) is likely to be one of the most innovative. It was initially proposed by Hammer et al. (2002) in the Falcon project for analysing the morphology of high-z galaxies. It was recently reconsidered in the phase A study of the multi-object integral field unit infrared (IR) spectrograph, called EAGLE (Cuby et al. 2010), in the frame of the European Extremely Large Telescope (Kissler-Patig 2010). MOAO is a key technology for this instrument (Rousset et al. 2010). Therefore, its development plan targets a pathfinder, called CANARY, with the goal of demonstrating on-sky the feasibility of MOAO (Myers et al. 2008).

MOAO aims at simultaneously compensating for the turbulence for a large number of very faint small science objects $\left(1-2^{\prime \prime}\right.$ each) spread over a wide field of view $\left(5-10^{\prime}\right)$ in a multiplex configuration. The disproportion between the size of the objects of interest and that of the field led to a concept of distributed $\mathrm{AO}$ corrections in MOAO, instead of a whole-field $\mathrm{AO}$ correction as in MCAO. Therefore, one DM per object is implemented in the optical train that feeds an integral field unit. In addition, the objects are generally too faint (such as the distant galaxies) for a measurement of the atmospheric distortions. It is therefore required to find a number of natural guide stars (NGS) in the wide field that are bright enough for this purpose. It is also envisioned to generate laser guide stars (LGS) when the number of available NGS is not sufficient (Rousset et al. 2010). Hence the wavefront measurements done across the field are mutually processed to reconstruct the information on the $3 \mathrm{D}$ turbulence volume by a tomographic approach (Ragazzoni et al. 1999). Then, the correction per object is computed by projection of the 3D turbulence volume in the object direction (Fusco et al. 2001; Vidal et al. 2010b). The main object in MOAO is the compensation made in open-loop for each object, i.e. with no feedback to the wavefront sensors (WFS) working on the NGS or/and LGS. Other side effects are noticeable, such as the difficulty of calibrating any optical relationship between the WFSs and DMs placed in different optical trains. A first on-sky testing of on-axis open-loop command has been reported (Andersen et al. 2008) as well as first MOAO laboratory demonstrations (Ammons et al. 2010; Vidal et al. 2010a).

CANARY's goal is to demonstrate on-sky the capability of driving a DM in open-loop by tomography using a number of 
Table 1. Three asterisms extracted from the Tycho 2 catalogue.

\begin{tabular}{c|cc|cc|cc|c}
\hline \hline$\#$ & Sep & $m_{V}$ & Sep & $m_{V}$ & Sep & $m_{V}$ & $m_{V}$ \\
\hline 47 & $47.9^{\prime \prime}$ & 9.9 & $40.6^{\prime \prime}$ & 10.2 & $53^{\prime \prime}$ & 8.7 & 11 \\
53 & $61.7^{\prime \prime}$ & 11.2 & $49.1^{\prime \prime}$ & 9.9 & $56.8^{\prime \prime}$ & 9.8 & 10.9 \\
12 & $39.3^{\prime \prime}$ & 11.2 & $31.4^{\prime \prime}$ & 10.7 & $51.5^{\prime \prime}$ & 10 & 8.3 \\
\hline
\end{tabular}

Notes. The columns indicate the CANARY reference number, the separation (in arcsec) of the off-axis stars from the central one, and the $V$ magnitudes of each.

in-the-field guide stars. This Letter briefly presents the instrument and the results obtained during a run of four nights in September 2010.

\section{CANARY demonstrator}

CANARY is designed to be set up at the 4.2-m William Herschel Telescope (WHT) at Roque de los Muchachos on La Palma, Canary Islands, Spain (Morris et al. 2010; Gendron et al. 2010). CANARY makes use of star asterisms formed by four NGSs. The central one, placed on-axis and used for diagnostic purpose, mimics the science object that will benefit from the turbulence compensation. We selected asterisms with a typical distance between the central on-axis star and the three off-axis ones ranging from 15 to $65^{\prime \prime}$, while keeping all stars brighter than $m_{V}=12$. The three observed asterisms of the 54 selected asterisms for the September period, are described in Table 1.

CANARY is installed at the Nasmyth focus of the WHT, where the field is optically de-rotated with a K-mirror. CANARY is equipped with three WFS that are able to patrol the telescope focal plane $\left(2.5^{\prime}\right.$ in diameter, plate scale $\left.0.22 \mathrm{~mm} /{ }^{\prime \prime}\right)$ and acquire the off-axis NGSs. The on-axis star is observed by a fourth WFS called Truth Sensor (TS), which measures the corrected wavefront after the DM. The four WFS are identical, they are Shack-Hartmann (SH) with $7 \times 7$ sub-apertures. They use Andor iXonEM 860 EMCCD cameras featuring $128 \times 128$ pixels. The measured read-out noise is $0.3,0.5,0.55$ and $0.7 \mathrm{e}^{-}$rms per pixel for the four cameras. We use $16 \times 16$ pixels of $0.25^{\prime \prime}$ scale in each SH sub-aperture, or any sub-window of it. The minimum distance between two off-axis WFSs is $20^{\prime \prime}$, limited by an anti-collision system. The central star beam is sent to two diagnostic tools via a two off-axis parabolae relay that includes a CILAS $8 \times 8$ piezostack array DM (conjugate to the pupil) and a high-speed tip-tilt mirror. At the output of the relay, the beam is split by a dichroic plate: the transmitted infrared flux (1.2 to $1.7 \mu \mathrm{m})$ is sent to the IR camera. This latter is a Xeva-1.7-320 from Xenics, featuring $320 \times 256$ pixels with a measured readout noise of $200 \pm 30 e^{-}$rms per pixel. The plate scale of the IR camera is $p=0.0371^{\prime \prime}$ per pixel. The camera has a single filter: $\mathrm{H}$ band. The visible part of the spectrum is reflected by the dichroic and goes to the TS. The TS allows us to check the turbulence compensation in real-time, to perform system calibration tasks, and to close the loop for performance comparison between conventional and open loop approaches. A telescope and source simulator allows us to completely characterise the system when under testing in the laboratory or during daytime maintenance at the telescope. The input focal plane of CANARY is also equipped with deployable sources (full-width at half-maximum either seeing or diffraction-limited). In addition, a specific feature is the so-called reverse-path calibration source, discussed in Sect. 3 .

The real-time computer (RTC) architecture is described in Dipper et al. (2010) and Basden et al. (2010). The system is driven at a selectable sampling frequency of up to $250 \mathrm{~Hz}$, which is limited by the WFS readout speed. The four WFS cameras are synchronised and read through dedicated FPGA-based acceleration hardware. The AO controller is based on CPU, featuring multi-core processors, and optimised for multi-threaded operation. The RTC exhibits $800 \mu$ s latency between the latest readout pixels of the WFSs and the time when the DM actuators are reaching half-stroke value. It also allows clients to obtain continuous or sub-sampled real-time data streams of images, slopes, and voltages. The RTC features a number of different real-time algorithms.

\section{Calibrations and algorithms}

The SH image processing algorithm uses an adaptive or fixed windowing of the SH spots on a sub-aperture-by-sub-aperture basis, together with centroiding done on thresholded spots or selected brightest pixels. These features allowed us to cope with the dynamics required for open-loop wavefront sensing depending on the observing conditions.

The linear tomographic reconstruction process is derived using the Learn $\mathcal{E}$ Apply algorithm from Vidal et al. (2010b), where the reconstructor is calibrated on-sky. Accumulating synchronous measurements with the three off-axis WFSs allows us to gather the input data of the reconstructor, while synchronous measurements from the TS form the expected outputs. The reconstructor is then found by solving - in a least-square sense the linear equation relating both inputs and outputs. In practice, data from all WFSs are recorded before observing (the grab phase of Learn), then data covariance matrices are computed and fitted to a model before being inverted to compute the reconstructor. The model involves the main parameters of the atmospheric turbulence profile and the NGS field positions, but also a variety of instrumental parameters such as the sensitivity of the WFSs, the slight pupil shifts, rotations, and magnifications that are left by residual errors in the optical alignment. Retrieving those parameters is important for tomographic reconstruction. They can either be retrieved by the fitting process from the recorded data themselves - for a perfect match, or they can be set to a given value when already calibrated - hence making the turbulence parameter fit more robust. As a pathfinder instrument, CANARY allows us to explore both options. In particular, it is equipped with the so-called reverse path calibration source, which is a seeing-limited source illuminating the DM from the relay output and feeding the light back into one of the open-loop WFS located in the input focal plane thanks to a retroreflector system, while preserving the pupil orientation. Therefore we can record interaction matrices with each off-axis WFS: they are the starting point for finding out all instrumental model parameters.

As in conventional AO (Boyer et al. 1990), those interaction matrices reflect the optical relationship between the DM and the WFSs. They are measured by actuating simultaneously all actuators with a sine wave pattern at a specific temporal frequency for each and retrieving the matrix thanks to a kind of spectral analysis. This particular method has been demonstrated to be the best one for identifying the open-loop DM model. The method has also demonstrated excellent on-sky capabilities with the TS: the quality of the on-sky determination of the non-zero matrix coefficients only differs by $2 \%$ from the laboratory ones.

The telescope focal plane contains field-variable static aberrations, possibly rotated by the optical de-rotator. Although it may be possible to model them, we preferred to follow a method where we measure them in situ. On the one hand, the on-sky recorded data from the off-axis WFSs (inputs for Learn) are 
averaged to estimate the static aberrations and used as reference slopes, i.e. subtracted from the off-axis measurements made during the observation. On the other hand, the average slope values of the on-axis TS, simultaneously measured, are subtracted from the TS previously calibrated close-loop reference slopes and translated into DM voltages $u_{\text {offset }}$ that will be applied as an offset on the DM while the open loop is engaged.

The open-loop controller is a temporal filter of the form $\boldsymbol{u}_{k+1}=(1-g) \boldsymbol{u}_{k}+g R \boldsymbol{s}_{k+1}$, where $\boldsymbol{u}_{k}$ is a voltage vector at iteration $k$, and $s_{k}$ the vector that concatenates the slopes of the three off-axis WFSs. The matrix $R$ is the tomographic reconstructor matrix, $g$ is the gain (scalar value, possibly different for DM and tip-tilt). The voltage actually applied on the DM is $\boldsymbol{v}_{k}=\boldsymbol{u}_{k}+u_{\text {offset }}$. For the closed-loop operation, we used the conventional integrator temporal controller (Gendron \& Léna 1995).

\section{Observations and data reduction}

We focus in this Letter on the results obtained on the fourth night of our observation (2010 Sep. 27). We alternated the observations between the SCAO (single-conjugate AO, stands for conventional AO in closed loop on the TS), GLAO and MOAO modes as the turbulence profile evolved and as we changed asterisms. We also collected external SLODAR (Wilson 2002) and DIMM (Sarazin \& Roddier 1990) data in parallel, which will be processed later along with our data. During the whole night, the sampling frequency of all WFSs was $150 \mathrm{~Hz}$ irrespective of the AO mode. Although the temporal controllers are slightly different between open and closed loop, the RTC latency is the same for all modes.

The IR images were subtracted from the background and compensated for dead pixels with a dead pixel map established on a dark image. All images were obtained using individual exposure times of 1 second, and sums of 30 images were saved. Strehl ratios (SR) have been computed on the IR images by normalizing their total energy to unity, and dividing their peak value by that of the diffraction-limited pattern sampled identically. This peak value is given by $a=\frac{\pi}{4}\left(D^{2}-o^{2}\right) p^{2} / \lambda^{2}$, with $o$ the central obscuration diameter, and $p$ the camera pixel scale. The normalization of the flux is dramatically sensitive to the estimation of background level to be subtracted. Special care has been taken with this operation, first narrowing the field to only $64^{2}$ pixels around the source and then estimating the residual background level at the periphery. We estimate the uncertainty on the SR to be of the order of 0.02 .

In parallel to the IR images, the real-time slopes of all WFSs (off-axis and TS) and DM voltage data were saved to determine the atmospheric parameters and evaluate the error budget. The performance was estimated from the TS by reconstructing the residual phase on the Zernike basis using modes $Z_{2}$ to $Z_{36}$ (Noll 1976) and computing the variance $\sigma_{\mathrm{TS}}^{2}=\sum_{2}^{36}\left\langle a_{i}^{2}\right\rangle$. The noise variance on the measured slopes of the TS is estimated by computing the temporal autocorrelation of each slope signal. The Dirac-like autocorrelation of the noise is separated from the smooth autocorrelation of turbulence by extrapolating the first points at the origin with a parabolic fit (Gendron \& Léna 1995). The TS wavefront noise values $\sigma_{\text {noise }}$ reported in the next section, expressed in $\mathrm{nm} \mathrm{rms}$, are the slope noise propagated through the Zernike reconstruction matrix on the 35 modes, assuming no spatial correlation of slope noise (diagonal covariance matrix). Hence, the AO loop residual error variance, as measured by the TS, is given by $\sigma_{\mathrm{TS}}^{2}-\sigma_{\text {noise }}^{2}$.

The value of $r_{0}$ is obtained with the open-loop off-axis WFSs. It is computed by fitting the theoretical variances of the

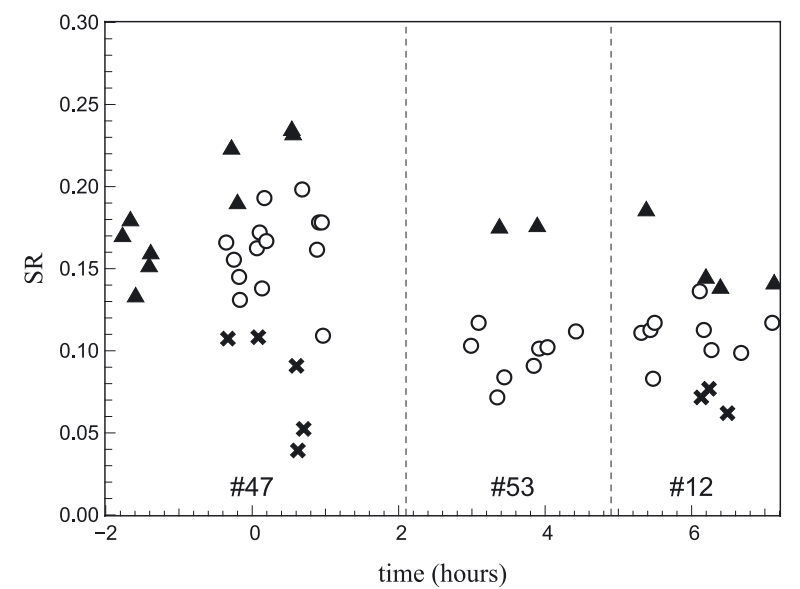

Fig. 1. Strehl ratios measured on the IR images ( $H$-band) versus local time (negative time before midnight) for the three asterisms. $\mathrm{SCAO}=\triangle$, MOAO $=\bigcirc$, GLAO $=\times$. The vertical dashed line indicates the change of asterism, labelled with their number.

Zernike decomposition of the Kolmogorov spectrum to those of the measured wavefront reconstructed on $Z_{4}$ to $Z_{36}$ (tip-tilt excluded from the fit). $r_{0}$ is given at the zenith angle of the observation and was not rescaled to zenith.

\section{Results}

The best SR in $H$-band obtained on-bench (no turbulence) is $0.70 \pm 0.02$, which corresponds to bench static aberration errors of the order of $\sigma_{\text {stat }}=150 \mathrm{~nm} \mathrm{rms}$, assuming the validity of $\mathrm{SR}=\exp \left(-\left(2 \pi \sigma_{\text {stat }} / \lambda\right)^{2}\right)$. Note that the best flat of the DM is $50 \mathrm{~nm} \mathrm{rms}$ (interferometric measurements). The on-bench SR is rather modest and the potential problem should be investigated further for the next runs.

$H$-band image SR are shown in Fig. 1 versus the local time. SCAO, MOAO and GLAO are respectively noted with triangles, circles, and squares. Although they correspond to different seeing and system configurations (loop gain and centroiding options, see Sect. 3), one can see that the MOAO performance lies between GLAO and SCAO ones. Note that the GLAO results presented here were achieved with the DM operating in open-loop, but with the full MOAO tomographic reconstructor replaced with a simple average of the off-axis WFS data. Note the relatively low performance of MOAO for asterism 53. This is because of the larger NGS separations, the increase of $C_{n}^{2}(h)$ at high altitude and a less accurate estimation of the field static aberrations when compared to the results with asterism 47 .

Figure 2 shows the same SR values obtained from the IR images, versus $r_{0}$ as deduced from the off-axis WFS data (Sect. 4): the different performance regimes clearly appear. As expected, the general trend is an increase of SR with the increase of $r_{0}$. The improvement from GLAO to MOAO shows the impact and the effectiveness of the tomographic reconstruction. We emphasize that points significantly scatter around an average behaviour, because they correspond to a variety of observing configurations. In addition, Fig. 2 mixes data taken on different asterisms, distances from zenith, tomographic reconstructors and $C_{n}^{2}(h)$ profiles. The detailed analysis that is required to distinguish all the effects will be provided in a subsequent paper.

The total variance of the on-axis residual wavefront is computed by $\sigma_{\phi}^{2}=\sigma_{\text {TS }}^{2}-\sigma_{\text {noise }}^{2}+\sigma_{\text {fit }}^{2}+\sigma_{\text {stat }}^{2}$. The corresponding SR, as deduced from the TS, is computed by $\mathrm{SR}_{\mathrm{TS}}=\exp \left(-\left(2 \pi \sigma_{\phi} / \lambda\right)^{2}\right)$. 


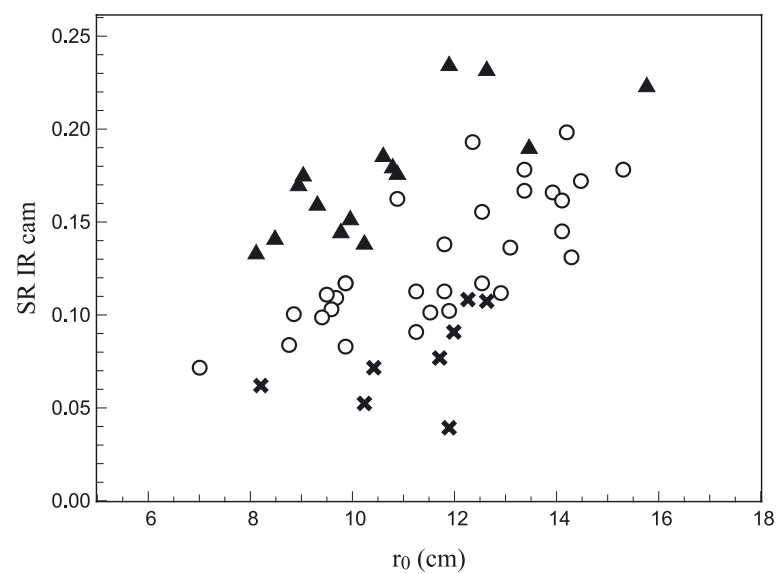

Fig. 2. Strehl ratios in $H$-band, same as in Fig. 1 , versus $r_{0}(0.5 \mu \mathrm{m})$ measured by the off-axis WFSs simultaneously with the IR images. SCAO = $\triangle, \mathrm{MOAO}=\bigcirc, \mathrm{GLAO}=\times$.

Table 2. Error budget (in nm rms).

\begin{tabular}{lccc}
\hline \hline & MOAO & MOAO & SCAO \\
& Oh10 & Oh11 & Oh32 \\
\hline average $r_{0}(\mathrm{~cm})$ & 12.3 & 13.3 & 11.9 \\
\hline TS measured $\sigma_{\mathrm{TS}}(\mathrm{nm})$ & 292 & 290 & 275 \\
noise error $\sigma_{\text {noise }}(\mathrm{nm})$ & 178 & 170 & 186 \\
\hline AO loop error $(\mathrm{nm})$ & 231 & 235 & 203 \\
fitting error $\sigma_{\text {fit }}(\mathrm{nm})$ & 174 & 163 & 179 \\
bench static aberr. $\sigma_{\text {stat }}(\mathrm{nm})$ & 150 & 150 & 150 \\
\hline Total $\sigma_{\phi}(\mathrm{nm})$ & 326 & 323 & 309 \\
\hline SR $)$ & 0.214 & 0.22 & 0.25 \\
IR camera SR $(H$ band $)$ & 0.21 & 0.193 & 0.244 \\
\hline
\end{tabular}

Notes. Comparison between the SR deduced from the TS slope data (14 s data) and the SR of the IR images.

The estimation of the DM fitting error $\sigma_{\text {fit }}$ (or undermodelling error) has been derived from the measured value of $r_{0}$ using the Noll formula for 36 compensated Zernike modes (Noll 1976). We have chosen three images with associated slope data to illustrate the wavefront error budget. Table 2 shows the results for two MOAO cases compared to a SCAO case. All files are taken on the same asterism (asterism 47) in fairly similar seeing conditions and system configuration. One can see that the main contributor to the wavefront error is the term called AO loop error, which is measured by the TS after the quadratic subtraction of the noise contributor. This term includes both static and dynamic contributors. It contains mainly temporal errors in SCAO mode, while in MOAO mode it additionally accounts for the tomographic and open-loop errors. Both cases also contain a contribution from noise propagated through the AO control: SCAO injects the TS noise in closed-loop, while MOAO injects in open loop the noise from the three off-axis WFSs propagated through the tomographic reconstructor. In the particular case of asterism 47, the SCAO noise is higher than the MOAO one (one star of 11 th magnitude against three stars 1 to 2 mag brighter, see Table 1). The detail of the error budget is a tricky problem and is left for the forthcoming paper. We can already see that the results of MOAO are similar to those of SCAO. This very preliminary attempt of drawing an error budget produces numbers that are consistent with the SR values that were observed on the IR camera. However, the exponential formula should be pessimistic in estimating $\mathrm{SR}_{\mathrm{TS}}$, leading to a few tens of nanometers of error that require further investigation.

\section{Conclusion}

MOAO is a new challenging AO technique that is optimised to perform simultaneous observations of a sparsely-populated objects within a very wide field of view, such as observations of distant galaxies in the early universe.

We report in this Letter how this technique has been successfully demonstrated on-sky for the first time by the CANARY demonstrator installed at the WHT in the Canary Islands, Spain. In CANARY, the MOAO uses in open loop three WFSs on three widely off-axis NGSs to compute by tomography the atmospheric turbulence real-time compensation delivered in open loop by the DM to the on-axis target. A preliminary analysis of the first on-sky results in September 2010 shows that MOAO nearly performs as well as conventional closed-loop adaptive optics. Strehl ratios of the order of 0.20 were observed in MOAO mode, compared to 0.25 in classical closed-loop AO mode in similar observing conditions $\left(r_{0}(0.5 \mu \mathrm{m})\right.$ around $\left.15 \mathrm{~cm}\right)$. The indepth analysis of the recorded data will be published in a forthcoming paper. The next step in the CANARY programme will be to perform the on-sky test of MOAO using four Rayleigh LGSs in addition to NGSs.

Acknowledgements. This work is supported by Agence Nationale de la Recherche (ANR) program 06-BLAN-0191, CNRS/INSU, Obs. de Paris, and Univ. Paris Diderot - Paris 7 in France, Science and Technology Facilities Council, Univ. of Durham in UK and European Commission FP7 (E-ELT Prep. Infrastruct. 2007-1 Grant 211257 and the program OPTICON JRA1).

\section{References}

Ammons, S. M., Johnson, L., Laag, E. A., et al. 2010, PASP, 122, 573

Andersen, D. R., Fischer, M., Conan, R., Fletcher, M., \& Véran, J. 2008, in Adaptive Optics Systems, Proc. SPIE, 7015

Basden, A., Geng, D., \& Myers, R. 2010, Applied Optics, 49, 6354

Boyer, C., Michau, V., \& Rousset, G. 1990, in Amplitude and Intensity Spatial Interferometry, Proc. SPIE, 1237, 406

Clénet, Y., Conan, J., Fusco, T., \& Rousset, G. (eds.) 2010, in 1st Adaptive Optics for Extremely Large Telescopes Conf. (EDP Sciences), 00001

Cuby, J., Morris, S., Fusco, T., et al. 2010, in Ground-based and Airborne Instrumentation for Astronomy III, Proc. SPIE, 7735

Dipper, N. A., Basden, A., Looker, N. E., et al. 2010, in Adaptive Optics Systems II, Proc. SPIE, 7736

Fusco, T., Conan, J., Rousset, G., Mugnier, L. M., \& Michau, V. 2001, JOSA A, 18,2527

Gendron, E., \& Léna, P. 1995, A\&AS, 111, 153

Gendron, E., Morris, T., Hubert, Z., et al. 2010, in Adaptive Optics Systems II, Proc. SPIE, 7736

Hammer, F., Sayède, F., Gendron, E., et al. 2002, in Scientific Drivers for ESO Future VLT/VLTI Instrumentation, ed. J. Bergeron, \& G. Monnet, 139

Kissler-Patig, M. 2010, in 1st Adaptive Optics for Extremely Large Telescopes Conf. (EDP Sciences), 01001

Marchetti, E., Brast, R., Delabre, B., et al. 2008, in Adaptive Optics Systems, Proc. SPIE, 7015

Milton, N. M., Lloyd-Hart, M., Baranec, C., et al. 2008, in Adaptive Optics Systems, Proc. SPIE, 7015

Morris, T., Hubert, Z., Myers, R., et al. 2010, in 1st Adaptive Optics for Extremely Large Telescopes Conf. (EDP Sciences), 08003

Myers, R. M., Hubert, Z., Morris, T. J., et al. 2008, in Adaptive Optics Systems, Proc. SPIE, 7015

Noll, R. J. 1976, JOSA, 66, 207

Ragazzoni, R., Marchetti, E., \& Rigaut, F. 1999, A\&A, 342, L53

Rousset, G., Fontanella, J. C., Kern, P., Gigan, P., \& Rigaut, F. 1990, A\&A, 230, L29

Rousset, G., Fusco, T., Assémat, F., et al. 2010, in Adaptive Optics Systems II, Proc. SPIE, 7736

Sarazin, M., \& Roddier, F. 1990, A\&A, 227, 294

Vidal, F., Gendron, E., Brangier, M., et al. 2010a, in 1st Adaptive Optics for Extremely Large Telescopes Conf. (EDP Sciences), 07001

Vidal, F., Gendron, E., \& Rousset, G. 2010b, JOSA A, 27, A253

Wilson, R. W. 2002, MNRAS, 337, 103 